\title{
K-Ras Plasma Membrane Interactions: A Tractable Therapeutic Target
}

\author{
Karen M Henkels ${ }^{1}$ and Kwang-jin Cho ${ }^{1 *}$ \\ ${ }^{1}$ Department of Biochemistry and Molecular Biology, School of Boonshoft Medical School, Wright State University, USA
}

*Corresponding author: Kwang-jin Cho, Department of Biochemistry and Molecular Biology, School of Boonshoft Medical School, Wright State University, Dayton, OH 45435, USA.

To Cite This Article: Kwang-jin Cho. K-Ras Plasma Membrane Interactions: A Tractable Therapeutic Target. 2020 - 9(5). AJBSR.MS.ID.001440.

DOI: 10.34297/AJBSR.2020.09.001440.

Received: 制June 30, 2020; Published: 盖 July 31, 2020

\begin{abstract}
Ras proteins are small GTPases that function like a molecular switch regulating cell proliferation, survival and differentiation at the plasma membrane (PM). Of the three major Ras isoforms, constitutively active mutations in K-Ras are frequently found in human cancers. Despite its critical role in tumorigenesis, no anti-K-Ras therapies are currently available in clinic. One strategy for blocking oncogenic K-Ras activity is to disrupt K-Ras interaction with the PM since K-Ras must primarily interact with the PM for its biological activity. This review will provide insights into recently reported molecular mechanisms that regulate K-Ras from the PM, which can be targeted to disrupt oncogenic K-Ras signaling.

Ras small GTPases are central regulators of cell proliferation, differentiation, survival and apoptosis. Consistent with this key regulatory role, constitutively active mutations of Ras are present in $\sim 19 \%$ of human cancers [1]. There are three ubiquitously expressed Ras isoforms in mammalian cells: $\mathrm{H}-, \mathrm{N}$ - and K-Ras (two splice variants, K-Ras4A and K-Ras4B), and mutations of the K-Ras isoform are found in $\sim 88 \%$ of pancreatic, $\sim 50 \%$ of colorectal, and $\sim 32 \%$ of all lung cancers [1]. Thus, anti-K-Ras therapies would have great clinical utility. There are four approaches currently being pursued for developing therapies for K-Ras-driven cancers: 1) dissociation of K-Ras from the plasma membrane (PM), 2) direct allosteric inhibition of K-Ras, 3) inhibition of K-Ras downstream effectors, and 4) dysregulation of cell metabolism [2,3]. This review will focus on recent strategies for dissociating K-Ras from the PM, which results in inhibition of K-Ras signaling.
\end{abstract}

Phosphatidylserine Depletion from the inner Pm Leaflet Dissociates K-Ras from the PM and Blocks Oncogenic K-Ras Signaling

Many studies clearly demonstrate that Ras proteins must bind primarily to the inner leaflet of the PM for its biological activity and blocking Ras/PM interaction results in inhibition of Ras signaling $[4,5]$. For a stable PM interaction, newly synthesized Ras proteins must undergo a series of post-translational modifications at the C-terminal CAAX motif (where $\mathrm{C}=\mathrm{Cys}, \mathrm{A}=$ aliphatic amino acids, and $\mathrm{X}=$ Met or Ser). First, a cytosolic farnesyltransferase attaches a farnesyl group to the Cys, which allows Ras to bind to the cytosolic leaflet of the ER [6,7]. RCE1 (Ras converting CAAX endopeptidase 1) then removes the AAX tripeptide, followed by the methylation of the now C-terminal prenylated Cys by ICMT (isoprenylcysteine carboxyl methyltransferase) [8,6]. N-, H-, and K-Ras4A are further modified with the addition of palmitic acids on one or two other Cys residues near the prenylated Cys [7], allowing the stable localization to the PM. K-Ras4B (hereafter, K-Ras) has a single farnesyl lipid moiety preceded by six Lys residues, called a polybasic domain [9], and the strong positive charge of this polybasic domain allows $\mathrm{K}$-Ras to interact with anionic phospholipids in the PM through electrostatic interaction $[10,11]$.

Phosphatidylserine (PtdSer) is an anionic phospholipid enriched in the inner PM leaflet. Its charged head group confers a strong negative electrostatic potential to the cytosolic face of the 
PM. K-Ras interacts with PM PtdSer through the combined effects of the C-terminal polybasic domain and the farnesyl chain of the lipid anchor, which together provide high selectivity for PtdSer over other anionic phospholipids. Depletion of PM PtdSer dissociates $\mathrm{K}$-Ras from the PM and blocks oncogenic K-Ras signaling in vivo and in vitro [12, 13, 14, 15, and 16], suggesting its essential role in K-Ras biological activity. Several molecular mechanisms that maintain PtdSer enrichment at the inner PM leaflet have been reported.

\section{Perturbation of sphingomyelin metabolism depletes PM PtdSer content}

Recent studies have demonstrated that acid and neutral sphingomyelinases (SMases) that convert sphingomyelin to ceramide in the lysosome and ER, respectively, regulate the PM localization of PtdSer and K-Ras. Acid SMase inhibitors including tricyclic anti-depressants mislocalize PtdSer and K-Ras from the PM to endo membranes and block oncogenic K-Ras signaling [13,16,17]. Replenishing PM PtdSer with exogenous PtdSer supplementation in these cells restores K-Ras PM binding and signaling, suggesting that the K-Ras PM mislocalization is through PtdSer depletion at the inner PM leaflet $[13,16,17]$. Furthermore, supplementation with recombinant Acid SMase returns PtdSer and K-Ras to the PM in Acid SMase-inhibited cells, suggesting that Acid SMase regulates PtdSer localization at the PM, resulting in K-Ras PM interaction [16]. In addition, inhibition of neutral SMase mislocalizes PtdSer and K-Ras from the PM and blocks oncogenic K-Ras signaling, and supplementation with exogenous PtdSer in these cells restores K-Ras PM localization [18]. Taken together, these studies suggest that acid and neutral SMases regulate oncogenic K-Ras signaling through maintaining PtdSer enrichment at the inner PM leaflet, and that acid and neutral SMases are tractable targets for the development of anti-K-Ras therapies.

\section{Phosphatidylinositol 4-phosphate regulates PtdSer transport from the ER to the PM}

Phosphatidylinositol 4-phosphate (PI4P) is synthesized from $\mathrm{PI}$ at the PM and Golgi complex by four PI 4-kinases in mammalian cells: PI4K II $\sim$ and $\therefore$ (PI4K2A and 2B), and PI4K III $\dot{\sim}$ and $\cdots$ (PI4KA and PI4KB) [19]. PI4KA and 2B are predominantly localized to the PM, while PI4K2A and PI4KB localize to the Golgi complex [19]. Recent studies have reported that PI4P content at the PM and Golgi complex regulate the PM localization of PtdSer and K-Ras. In mammalian cells, oxysterol-binding protein-related protein (ORP) 5 and 8 are recruited to ER-PM membrane contacting sites (MCSs) to exchange newly synthesized PtdSer from the ER for PI4P from the PM $[20,21]$.

This exchange is driven by PM PI4P synthesis by PI4KA and the concomitant hydrolysis of PI4P by Sac1 phosphatase in the ER to maintain a PI4P gradient across the PM and ER. Eliminating any component of this machinery depletes PtdSer content at the inner PM leaflet and mislocalizes K-Ras from the PM [22, 23]. PI4KA inhibition further blocks growth of multiple K-Ras-driven cancer cells in vitro [23]. Moreover, Golgi PI4P depletion by glucose starvation or PI4KB knockout redistributes PtdSer from the PM to endomembranes, resulting in K-Ras translocation to mitochondria and inhibited oncogenic K-Ras signaling [24]. These studies suggest that molecular mechanisms that maintain PI4P content at the PM and Golgi complex is a plausible target for blocking oncogenic K-Ras signaling.

\section{K-Ras phosphorylation Dissociates K-Ras from the PM and Abrogates Oncogenic K-Ras Signaling}

\section{Protein kinase C (PKC) phosphorylates K-Ras blocking oncogenic K-Ras signaling}

Another mechanism to dissociate K-Ras from the PM and block oncogenic K-Ras signaling is K-Ras phosphorylation by PKC. PKC directly phosphorylates K-Ras at Ser181 and to a lesser extent at Ser171 and Thr183, redistributing K-Ras from the PM to the endomembranes including mitochondria, where it triggers enhanced apoptosis [25]. PKC activators can further suppress the growth of $\mathrm{K}$-Ras tumors in nude mice by stimulating K-Ras phosphorylation $[25,26]$. A recent study further demonstrated that chalcone-based small molecules stimulate PKC, which phosphorylates K-Ras at Ser181, resulting in K-Ras PM dissociation and inhibition of K-Rasdriven human cancer cell growth [27]. There are three classes of PKC isozymes: conventional $(\alpha, \beta, \gamma)$, novel $(\delta, \varepsilon, \eta, \theta)$, and atypical $(\zeta, \mathrm{l})$. Several studies propose that $\operatorname{PKC} \delta$ is a suppressor for oncogenic K-Ras. PKC $\delta$ protein levels are lower in endometrial cancer cells harboring oncogenic mutant K-Ras than WT K-Ras [28], and approximately $40 \%$ of $\mathrm{PKC} \delta$ loss-of-function mutations are found in pancreatic cancers harboring oncogenic mutant K-Ras [29]. Moreover, patients with non-small cell lung cancers expressing oncogenic mutant K-Ras show an increased overall survival rate when they also have higher PKC $\delta$ mRNA levels [30]. Taken together, these studies suggest that PKC $\delta$ may have an anticancer activity in cancers expressing oncogenic mutant K-Ras possibly by dissociating oncogenic mutant K-Ras from the PM and thereby, abrogating oncogenic K-Ras signaling.

\section{Stimulating the AMPK/eNOS/PKG2 pathway phosphorylates K-Ras, blocking oncogenic K-Ras signaling}

In addition to PKC, cGMP-dependent protein kinase 2 (PKG2) has been identified as a new K-Ras kinase phosphorylating K-Ras at Ser181 through the AMPK/eNOS/PKG2 signaling pathway [13]. A recent study demonstrated that direct or indirect activation of AMP-activated protein kinase (AMPK) stimulates the activity of endothelial nitric oxide synthase (eNOS), one of the AMPK 
downstream effectors. This in turn, elevates cellular nitric oxide levels, which promotes soluble guanylyl cyclase (sGC) activity, generating cGMP from GTP. Upon cGMP binding, activated PKG2 $[31,32]$, but not PKG1, is recruited to the PM and phosphorylates K-Ras at Ser181, resulting in K-Ras dissociation from the PM. Moreover, stimulation of the AMPK/eNOS/PKG pathway shows anti-cancer activity. Chronic activation of components in the signaling pathway by pharmacological agents inhibits the growth of non-small cell lung cancer cells expressing oncogenic mutant K-Ras [13].

\section{Conclusion}

Oncogenic mutant K-Ras is found in approximately $14 \%$ of all human cancers, but no anti-K-Ras therapies are available in clinic. Despite the critical role of K-Ras interaction with the PM for its biological activity, the exact molecular mechanisms of K-Ras transport to and maintenance at the PM are not fully understood. Investigating these mechanisms will provide insights into novel approaches of developing anti-K-Ras therapeutics.

\section{Acknowledgement}

This work was supported by the National Cancer Institute [R00-CA188593 to K.-J.C.].

\section{Reference}

1. Prior I A, Hood F E, Hartley J L (2020) The frequency of Ras mutations in cancer. Cancer Res.

2. Gorfe A A, Cho K J (2019) Approaches to inhibiting oncogenic K-Ras. Small GTPases 1-10.

3. Papke, B, Der C J (2017) Drugging RAS: Know the enemy. Science 355(6330): 1158-1163.

4. Hancock J F (2003) Ras proteins: different signals from different locations. Nat Rev Mol Cell Biol 4(5): 373-384

5. Willumsen B M, Christensen A, Hubbert N L, Papageorge A G, Lowy D R, et al. (1984) The p21 ras C-terminus is required for transformation and membrane association. Nature 310(5978): 583-586.

6. Gutierrez L, Magee A I, Marshall C J, Hancock J F (1989) Post-translational processing of p21ras is two-step and involves carboxyl-methylation and carboxy-terminal proteolysis. EMBO J 8(4): 1093-1098.

7. Hancock J F, Magee A I, Childs J E, Marshall C J (1989) All ras proteins are polyisoprenylated but only some are palmitoylated. Cell 57(7): 11671177.

8. Clarke S, Vogel J P, Deschenes R J, Stock J (1988) Posttranslationa modification of the Ha-ras oncogene protein: evidence for a third class of protein carboxyl methyltransferases. Proc Natl Acad Sci U S A 85(13): 4643-4647.

9. Hancock J F, Paterson H, Marshall C J (1990) A polybasic domain or palmitoylation is required in addition to the CAAX motif to localize p21ras to the plasma membrane. Cell 63(1): 133-139.

10. Yeung T, Gilbert G E, Shi J, Silvius J, Kapus A, et al. (2008) Membrane phosphatidylserine regulates surface charge and protein localization. Science 319(5860): 210-213.

11. Cho K J, van der Hoeven D, Zhou
12. Cho K J, Park J H, Piggott A M, Salim A A, Gorfe A A, et al. (2012) Staurosporines disrupt phosphatidylserine trafficking and mislocalize Ras proteins. J Biol Chem 287(52): 43573-43584.

13. Cho K J, Casteel D E, Prakash P, Tan L, van der Hoeven D, et al. (2016) AMPK and Endothelial Nitric Oxide Synthase Signaling Regulates K-Ras Plasma Membrane Interactions via Cyclic GMP-Dependent Protein Kinase 2. Mol Cell Biol 36(24): 3086-3099.

14. Tan L, Cho K J, Kattan W E, Garrido C M, Zhou Y, et al. (2019) Acylpeptide hydrolase is a novel regulator of KRAS plasma membrane localization and function. J Cell Sci 132(15): jcs232132.

15. Tan L, Cho K J, Neupane P, Capon R J, Hancock F, et al. (2018) An oxanthroquinone derivative that disrupts RAS plasma membrane localization inhibits cancer cell growth. J Biol Chem 293(35): 1369613706.

16. van der Hoeven D, Cho K J, Ma X, Chigurupati S, Parton R G, et al. (2013) Fendiline inhibits K-Ras plasma membrane localization and blocks K-Ras signal transmission. Mol Cell Biol 33(2): 237-251.

17. van der Hoeven D, Cho K J, Zhou Y, Ma X, Chen W, et al. (2018) Sphingomyelin Metabolism Is a Regulator of K-Ras Function. Mol Cell Biol 38.

18. Garrido C M, Henkels K M, Rehl K M, Liang H, Zhou Y, et al. (2020) Avicin $\mathrm{G}$ is a potent sphingomyelinase inhibitor and blocks oncogenic $\mathrm{K}$ - and H-Ras signaling. Sci Rep 10: Pp 9120.

19. Balla T (2013) Phosphoinositides: tiny lipids with giant impact on cell regulation. Physiol Rev 93(3): 1019-1137.

20. Chung J, Torta F, Masai K, Lucast L, Czapla H, et al. (2015) INTRACELLULAR TRANSPORT. PI4P/phosphatidylserine countertransport at ORP5- and ORP8-mediated ER-plasma membrane contacts. Science 349(6246): 428-432.

21. Moser von Filseck J, Copic A, Delfosse V, Vanni S, Jackson C L, et al. (2015) INTRACELLULAR TRANSPORT. Phosphatidylserine transport by ORP/ Osh proteins is driven by phosphatidylinositol 4-phosphate. Science 349(6246): 432-436

22. Gulyas G, Radvanszki G, Matuska R, Balla A, Hunyady L, et al (2017) Plasma membrane phosphatidylinositol 4-phosphate and 4,5-bisphosphate determine the distribution and function of K-Ras4B but not H-Ras proteins. J Biol Chem 292(46): 18862-18877.

23. Kattan W E, Chen W, Ma X, Lan T H, van der Hoeven D, et al. (2019) Targeting plasma membrane phosphatidylserine content to inhibit oncogenic KRAS function. Life Sci Alliance 2(5): e201900431.

24. Miller T E, Henkels K M, Huddleston M, Salisbury R, Hussain S M, et al. (2019) Depletion of phosphatidylinositol 4-phosphate at the Golgi translocates K-Ras to mitochondria. J Cell Sci 132(16): jcs231886.

25. Bivona T G, Quatela S E, Bodemann B O, Ahearn I M, Soskis MJ, et al. (2006) PKC regulates a farnesyl-electrostatic switch on K-Ras that promotes its association with Bcl-XL on mitochondria and induces apoptosis. Mol Cell 21(4): 481-493.

26. Wang M T, Holderfield M, Galeas J, Delrosario R, To M D, et al. (2015) K-Ras Promotes Tumorigenicity through Suppression of Non-canonical Wnt Signaling. Cell 163(5): 1237-1251.

27. Kovar S E, Fourman C, Kinstedt C, Williams B, Morris C, et al. (2020) Chalcones bearing a 3,4,5-trimethoxyphenyl motif are capable of selectively inhibiting oncogenic K-Ras signaling. Bioorg Med Chem Lett 30(11): 127144.

28. Reno E M, Haughian J M, Dimitrova I K, Jackson T A, Shroyer K R, (2008) Analysis of protein kinase C delta (PKC delta) expression in endometrial tumors. Hum Pathol 39(1): 21-29.

29. Antal C E, Hudson A M, Kang E, Zanca C, Wirth C, et al. (2015) Cancerassociated protein kinase $\mathrm{C}$ mutations reveal kinase's role as tumor suppressor. Cell 160(3): 489-502. 
30. Ohm A M, Tan A C, Heasley L E, Reyland M E (2017) Co-dependency of PKCdelta and K-Ras: inverse association with cytotoxic drug sensitivity in KRAS mutant lung cancer. Oncogene 36(30): 4370-4378.

31. Y, Maekawa M, Ma X, et al. (2015) Inhibition of Acid Sphingomyelinase Depletes Cellular Phosphatidylserine and Mislocalizes K-Ras from the Plasma Membrane. Mol Cell Biol 36(2): 363-374.
32. Zhou Y, Prakash P, Liang H, Cho K J, Gorfe A A, et al. (2017) Lipid-Sorting Specificity Encoded in K-Ras Membrane Anchor Regulates Signal Output. Cell 168(1-2): 239-251. 